Case Report

\title{
PATIENT UNDERGOING COMBINED RIGHT RADICAL NEPHRECTOMY AND RIGHT ATRIAL TUMOUR EXCISION : ANAESTHETIC MANAGEMENT
}

\author{
Manjunath R. Kamath ${ }^{1}$, Krishna Prasad $\mathrm{P}^{1}$, Mundayat Gopalakrishnan ${ }^{2}$, \& Rajeev T.P. ${ }^{3}$ \\ ${ }^{1}$ Department of Anaesthesia, ${ }^{2}$ Cardiothoracic and Vascular Surgery, ${ }^{3}$ Urology. \\ Justice K.S. Hegde Charitable Hospital, Nitte University, Mangalore, Karnataka - 575 018, India \\ Correspondence: \\ Associate Professor and Consultant Cardiac Anaesthesiologist, K.S. Hegde Medical Academy, \\ Deralakatte, Mangalore - 575018 \\ Mobile : 98454 12094, E-mail : manjunathrkamath@gmail.com
}

\section{Abstract :}

We describe anaesthetic management of a rare case of right atrial tumour with right renal mass posted for combined excision of right atrial tumor and right radical nephrectomy. Anaesthesia plan was combined thoracic epidural anaesthesia \& general anaesthesia. Right renal mass was removed through the anterior subcostal incision, followed by the right atrial mass excision through the midline sternotomy incision under cardiopulmonary bypass. Issues related to the possibility of inferior vena cava (IVC) thrombus \& its extension into the right atrium and the subsequent removal, pain management, ideal position of the central venous access, cardiopulmonary bypass with a single kidney are discussed.

Keywords: Atrial myxoma, Renal cell carcinoma (RCC), IVC thrombus, Cardiopulmonary bypass (CPB)

\section{Introduction :}

Myxoma is the common benign intracardiac tumor in adults accounting for nearly $40 \%$ of all cardiac tumors. ${ }^{1} \mathrm{~A}$ far more common cause of right atrial tumors are metastatic tumors that arise in the abdomen and pelvis, especially renal cell carcinoma, which directly invade the inferior vena cava and extend into the right heart. Tumor-associated thrombus, degeneration, hemorrhage, calcification, inflammatory infiltrate may lead to an incorrect diagnosis of mural thrombi or endocardial vegetations. Radical operations for tumor extension into the right atrium requires careful planning. ${ }^{2}$ Misinterpretation of a cardiac mass may result in an incorrect management strategy and therefore accurate diagnosis is crucial.

\section{Case Report :}

51 year old male patient presented with history of haematuria and dyspnoea on exertion. Computed Tomography (CT) abdomen revealed heterogeneously enhancing mass lesion in the upper pole of right kidney extending up to the calyces \& renal pelvis - s/o renal neoplasm. (figure 1 ) ECHO findings revealed an intracardiac mass in the right atrium attached to the right interatrial septum (figure 2) and CT thorax showed hypo-dense lesion in the right atrium and possibly right proximal part of pulmonary artery hinting at the possibility of myxoma/thrombus. (figure 3) He was posted for combined right radical nephrectomy and excision of right atrial tumor.

On the day of surgery, he was premedicated with oral diazepam $10 \mathrm{mg}$ and pantoprazole $20 \mathrm{mg}$ one $\mathrm{hr}$ before the commencement of surgery. Electrocardiography (ECG), pulse oximetry $\left(\mathrm{SpO}_{2}\right)$, end tidal capnography $\left(\mathrm{ETCO}_{2}\right)$, temperature, invasive blood pressure, central venous pressure (CVP) monitoring and Trans-oesophageal Echocardiography (TEE) (fig) were planned. Intravenous access was secured with $14 \mathrm{G}$ cannula in the right antecubital vein and arterial line with 206 cannula in the right radial artery.

$18 \mathrm{G}$ epidural catheter was then placed at T8-T9 inter-space and a test dose of $2 \mathrm{ml}$ of $2 \%$ lignocaine with adrenaline was injected. Epidural infusion of inj. bupivacaine $(0.2 \mathrm{mg} / \mathrm{ml})$ with inj. buprenorphine $(6 \mathrm{mcg} / \mathrm{ml})$ was started at 5 $\mathrm{ml} / \mathrm{hr}$.

General anesthesia was induced with fentanyl $350 \mathrm{mcg}$, midazolam $4 \mathrm{mg}$ \& profofol $50 \mathrm{mg}$ intravenously. Neuromuscular blockade was achieved with pancuronium 
7mg. The airway was secured with 8.5 Fr ID COETT. Central venous catheter ( $7 \mathrm{fr} 16 \mathrm{~cm}$ 3-lumen) was placed in the right internal jugular vein after intubation. Anaesthesia was maintained with oxygen in air $\left(\mathrm{FiO}_{2} 40 \%\right)$ and isoflurane 0.61.0 MAC supplemented with additional fentanyl, pancuronium and midazolam when necessary.

Right (Kocher's) anterior subcostal incision was performed and extended till post-axillary line laterally \& past midline medially. Right kidney was identified and mobilized. IVC and renal vein were examined for tumour extension and were found to be free. Renal artery and vein were ligated. Ureter was ligated, adrenal gland was separated \& kidney was removed (figure 4). Drain was placed in the retroperitoneal space, and the abdomen was closed in layers.

Midline sternotomy was then performed; CPB was established after cannulation of the aorta \& high cannulation of the SVC and IVC. RA was opened; huge tumour attached to septum was excised with the portion of the septum attached (figure 5). The defect was closed with a pericardial patch. Specimens were sent for histopathology. RA was then closed. Patient was weaned off cardiopulmonary bypass without any complications. Mediastinal and right pleural drains were placed. Atrial and ventricular pacing wires were inserted and chest was closed in layers.

Patient was extubated on the first postoperative day. Drains \& monitoring lines were removed by POD2; abdomen drains by POD4. Analgesia was maintained with epidural infusion of bupivacaine and buprenorphine. Patient was shifted to the ward on POD5.

Histopathology report revealed chromophobe renal cell carcinoma Grade 2 and right atrial myxoma.

\section{Discussion :}

The most common primary benign cardiac neoplasm is myxoma, about 15 to $20 \%$ of which arise in the right atrium $^{3}$. The diagnosis is usually established by echocardiography. Various normal and abnormal structures may appear as a mass in the right atrium on $2 \mathrm{D}$ echocardiography such as the vegetations, thrombi, Eustachian valve, pacemaker wire and central-line catheter. If the attachment site can be established, the diagnosis is almost assured because the majority of myxomas are attached to the interatrial septum, at the border of the fossa ovalis ${ }^{4}$.

But secondary tumors of the heart occur 40-50 times more frequently than primary cardiac tumors. Most common right atrial tumors are metastatic tumors like hepatoma and renal cell carcinoma . ${ }^{5,6}$ RCC is known to be the most lethal of all the genitourinary tumors. ${ }^{7}$

The most common factors influencing the prognosis are local infiltration to perinephric tissue, lymph node involvement, distant metastases, pathological stage of the tumor and invasion to the vena cava regardless of the level of tumor extension. ${ }^{8}$ Radical or partial nephrectomy remains the mainstay of curative treatment in case of patients with clinically localized and locally advanced disease ${ }^{9 .}$ But if the tumour is extending into the IVC and right atrium, the surgical approach should be individualized according to the level of the tumor thrombus and decision should be made by both cardiovascular surgeon and urologist. The choice of incision, caval control method, and venous return provision are important factors in surgical approach.

A combined subcostal incision with median sternotomy is the most appropriate method for adequate access to the kidneys, the IVC and the heart. The use of cardiopulmonary bypass improves control of blood loss, reduces the chance of pulmonary embolism and provides the necessary exposure to achieve complete clearance of the tumor thrombus from the IVC and right atrium. In some cases, hypothermic circulatory arrest allows complete resection of the tumor in a virtually bloodless operation field. ${ }^{10}$ However, it has potential renal, hepatic, neurological \& septic complications.

Therefore, complete staging should be performed for these patients. The role of MRI in the evaluation of the renal vein and IVC for detection of the thrombus extension is crucial. ${ }^{11}$ Another good technique for detection of the thrombus 


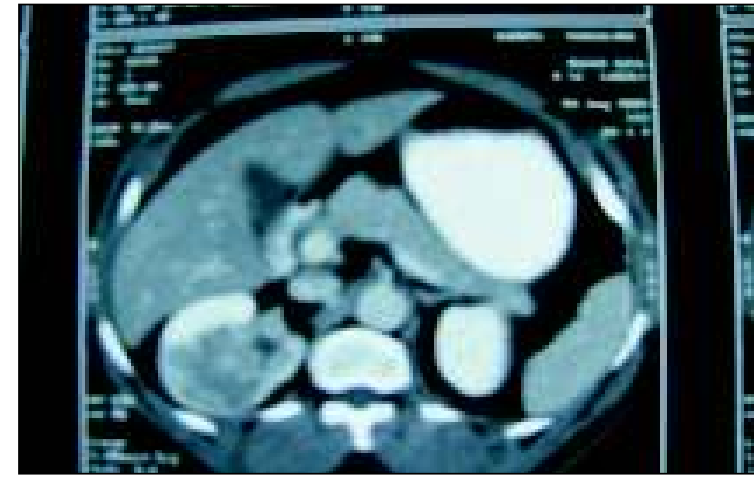

Figure 1 : C T abdomen: Heterogeneous enhancing mass lesion in the upper pole of right kidney extending up to the calyces \& renal pelvis - s/o renal neoplasm

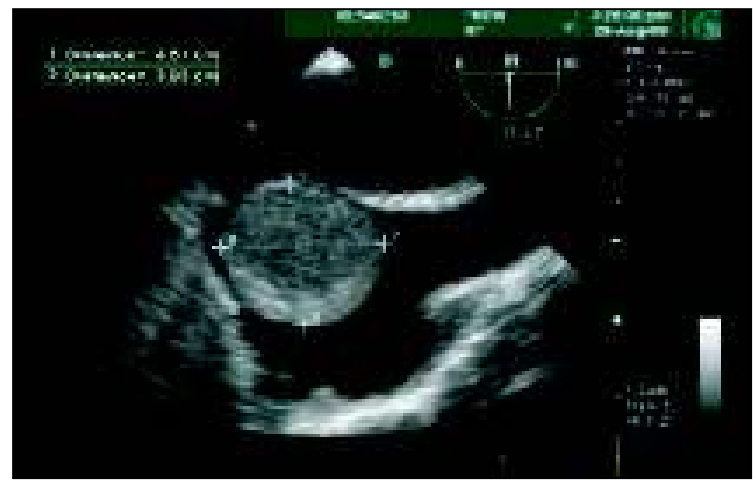

Figure 2 : ECHO: An intra cardiac mass in the right atrium attached to the right inter atrial septum.

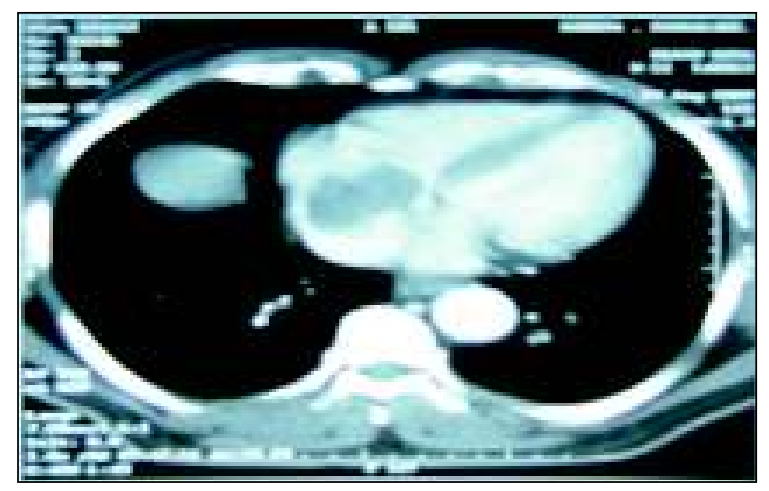

Figure 3 : C T CHEST: Hypo-dense non-enhancing lesion in the right atrium and right proximal part of pulmonary artery hinted at the possibility of myxoma/thrombus

extension into the right atrium is preoperative and intraoperative transesophageal echocardiography.

In our patient, CT thorax and abdomen showed clean IVC suggesting localized renal cell tumour and echocardiography showed right atrial tumour attached to inter-atrial septum suggesting myxoma. We planned right radical nephrectomy through anterior subcostal incision
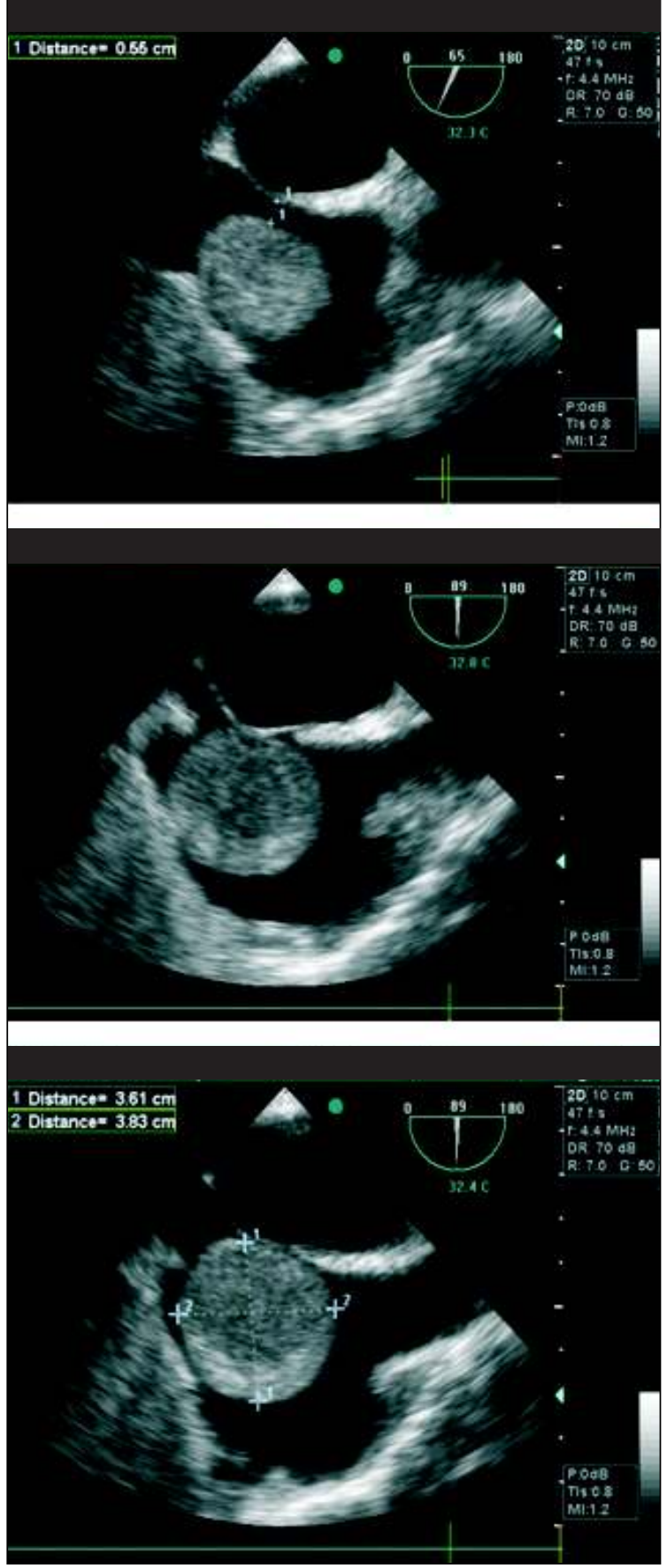

INTRAOPERATIVE TRANS-OESOPHAGEAL ECHO

first and then sternotomy and right atrial tumour excision on CPB.

Surgery involving both procedures in a same setting requires an extensive incision. So, epidural anaesthetic technique was employed in an attempt to improve patient comfort with less respiratory depression. At the same time it facilitated early extubation and discharge from ICU 


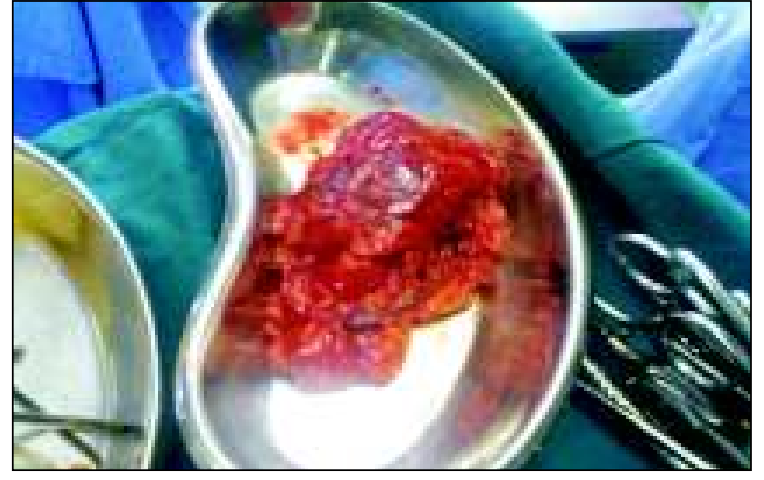

Figure 4 : Right renal mass

without compromising pain control.

Regardless of the route of insertion the optimal position of the tip of central venous catheter is the SVC junction with the right atrium. Right femoral vein can be cannulated for CVP monitoring to prevent dislodgement of right atrial mass if thrombus is suspected. But clamping of the inferior venacava during the excision of the renal mass can be troublesome. We cannulated right internal jugular vein under careful observation.

Single kidney impairs the ability to maintain body fluids,

\section{References:}

1. Bruke A, Virmani R. In: Tumors of the heart and great vessels: Atlas of Tumor Pathology. $3^{\text {rd }}$ series, fascicle 16.Washington, DC: Armed Forces Institute of Pathology 1995: 171-7.

2. Hatcher PA, Anderson EE, Paulson DF, Carson CC, Robertson JE. Surgical management and prognosis of renal cell carcinoma invading the vena cava. J. Urol. 1991; 145: 20-4.

3. Livi U, Bortolotti U, Milano A, Valente M, Prandi A Frugonic C, et al. Cardiac myxomas: results of 14 years' experience. Thorac Cardiovasc Surg. 1984; 32: 143-7.

4. Reynen K. Cardiac myxomas. N EnglJ Med 1995; 333: 1610-17.

5. Allen G, Klingman R, Ferraris VA, et al. Trans oesophageal echocardiography in the surgical management of renal cell carcinoma with intracardiac extension. J Cardiovasc Surg(Torino) 1991; 32(6): 833-6.

6. Van Camp G,Abdulsater J, Cosyns B, Liebens I,Vandenbossche JL.

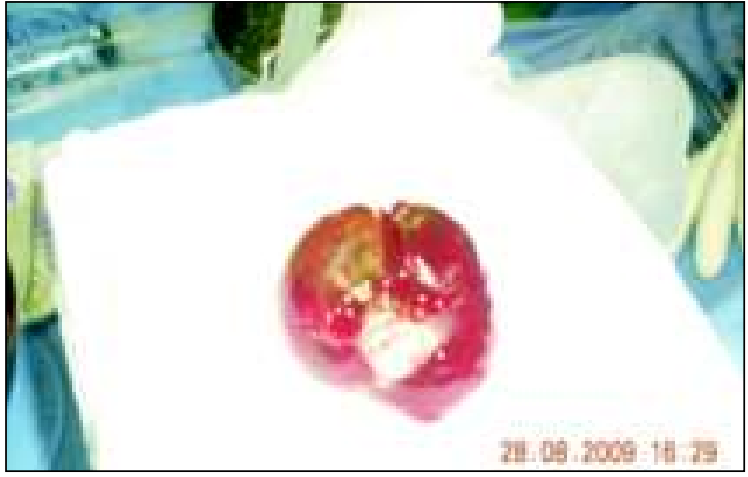

Figure 5 Right atrial tumour

electrolytes and acid base balance in the face of operative stress and helps to excrete waste products and drug metabolites. For patients undergoing cardiopulmonary bypass, particular concern exists because of the large fluid load typical with a crystalloid cardiopulmonary bypass prime and the potassium usually is administered as part of the cardioplegic solution. In our case, patient was weaned off bypass without any untoward events.

The increased risk of hemorrhage secondary to the heparinization should not be underestimated.

Transesophageal echocardiography of right atrial metastasis of a hepatocellular carcinoma. Chest 1994; 105(3): 945-7.

7. Mulders PF, Brouwers AH, Hulsbergen-van der Kaa CA, van Lin EN, Osanto S, de Mulder PH (February 2008). "[Guideline 'Renal cell carcinoma']" 152 (7): 376-80.

8. Blute ML, Leibovich BC, Lohse $\mathrm{CM}$, Cheville JC, Zincke $\mathrm{H}$. The Mayo Clinic experience with surgical management, complications and outcome for patients with renal cell carcinoma and venous tumour thrombus. BJU Int. 2004; 94:33-41.

9. Rini BI, Rathmell WK, Godley P (May 2008). "Renal cell carcinoma". Curr Opin Oncol 20 (3): 300-6.

10. Skinner DG, Pritchett TR, Lieskovsky G, Boyd SD, Stiles QR. Vena caval involvement by renal cell carcinoma. Surgical resection provides meaningful long-term survival. Ann Surg. 1989; 210:387-92.

11. Oto A, Herts BR, Remer EM, Novick AC. Inferior vena cava tumor thrombus in renal cell carcinoma: staging by MR imaging and impact on surgical treatment. AJR Am J Roentgenol. 1998; 171:1619-24. 